\title{
Teste Automático de Brückner basedo em Imagens
}

\author{
Italo F. S. Silva ${ }^{1}$, João D. S. Almeida ${ }^{1}$, Jorge A. M. Teixeira ${ }^{1}$, \\ Geraldo Braz Junior ${ }^{1}$, Anselmo C. de Paiva ${ }^{1}$ \\ ${ }^{1}$ Núcleo de Computação Aplicada - Universidade Federal do Maranhão (UFMA) \\ Av. dos Portugueses, 1966 - Bacanga - CEP 65080-805 - São Luís - MA

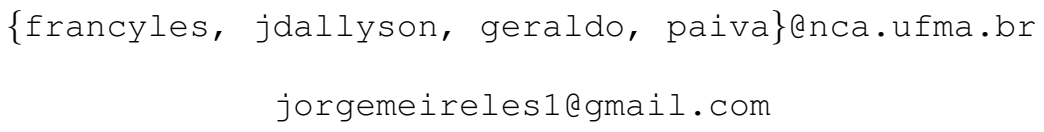

\begin{abstract}
Brückner Test is an important eye exam by witch it is possible to diagnose eye diseases early. This work presents a method to detect the presence of eye pathology based on images, using Haralick descriptors for texture analysis of the reflex and machine learning to classify normal and pathological cases. The proposed method reaches $91 \%$ accuracy, $90.9 \%$ sensibility and $91.14 \%$ specificity by using the REPTree classifier.
\end{abstract}

Resumo. O teste de Brückner é um importante exame oftalmológico pelo qual é possível diagnósticar doenças oculares precocemente. Este trabalho apresenta um método para detectar a presença de patologia na visão baseado em imagens, fazendo uso dos descritores de Haralick para analisar a textura do reflexo $e$ aprendizado de máquina para classificar os casos em normais ou patológicos. O método proposto alcança $91 \%$ de acurácia, 90,9\% de sensibilidade e 91,14\% de especificidade utilizando o classificador REPTree.

\section{Introdução}

O teste de Brückner é um importante exame oftalmológico através do qual é possível detectar patologias na visão precocemente. Também conhecido como exame do reflexo vermelho, esse teste pode ser usado como parte da rotina de exames de crianças recémnascidas, contribuindo, assim, para a identificação prematura de problemas oculares, incluindo a catarata congênita, erros refrativos e retinoblastoma [Sun et al. 2016].

Durante o exame, uma fonte luminosa incide luz nos olhos do paciente. Ao entrar em contato com a parte interna do olho, que é bastante irrigada por vasos sanguíneos, a luz é refletida na cor vermelha. Isso mostra que as principais estruturas internas do olho estão transparentes, permitindo que a luz atinja a retina normalmente. Entretanto, existem casos em que o reflexo não possui boa qualidade, pois, a luz é refletida com outra tonalidade ou é totalmente absorvida, caracterizando assim uma possível presença de patologia. $\mathrm{O}$ fenômeno do reflexo retiniano é muitas vezes observado em fotografias com flash.

O presente trabalho apresenta um método para detectar a presença de patologia na visão em imagens do teste automático de Brückner. A partir de fotografia e da utilização de técnicas de processamento de imagens e aprendizado de máquina, o método identifica se o olho está saudável ou com algum problema oftalmológico, auxiliando, dessa forma, a atuação profissional, facilitando e melhorando a qualidade da assistência. 
$\mathrm{Na}$ literatura, existem trabalhos que propõem métodos para a identificação de leucocoria, caracterizada pelo reflexo branco, utilizando a teoria de lógica fuzzy para combinar classificadores [Rivas-Perea et al. 2014] ou redes neurais convolucionais [Henning et al. 2014]. Esses trabalhos se limitam ao caso da leucocoria, desconsiderando outras patologias. O método proposto, ainda em desenvolvimento, em teste iniciais, utiliza os descritores de Haralick [Haralick et al. 1973] para extrair as características de textura e classifica as imagens em casos normais ou patológicos.

Este trabalho está organizado como segue: a Seção 2 descreve o método proposto. A Seção 3 mostra os resultados e sua análise e, por fim, a Seção 4 apresenta as conclusões obtidas com o experimento.

\section{Materiais e Métodos}

Esta seção apresenta os procedimentos realizados no desenvolvimento do método proposto neste trabalho, trazendo também informações sobre o conjunto de imagens utilizado para validar a proposta.

\subsection{Base de Imagens}

As imagens utilizadas no presente trabalho foram adquiridas de pacientes em uma clínica oftalmológia em São Luís-MA por meio de uma câmera com a função flash ativada. Alguns exemplos dessas imagens podem ser vistos na Figura 1. Os pacientes que se voluntariaram a participar do experimento assinaram um formulário de consentimento. Ressaltase também que esse estudo recebeu a aprovação do comitê de ética para o uso de imagens humanas.
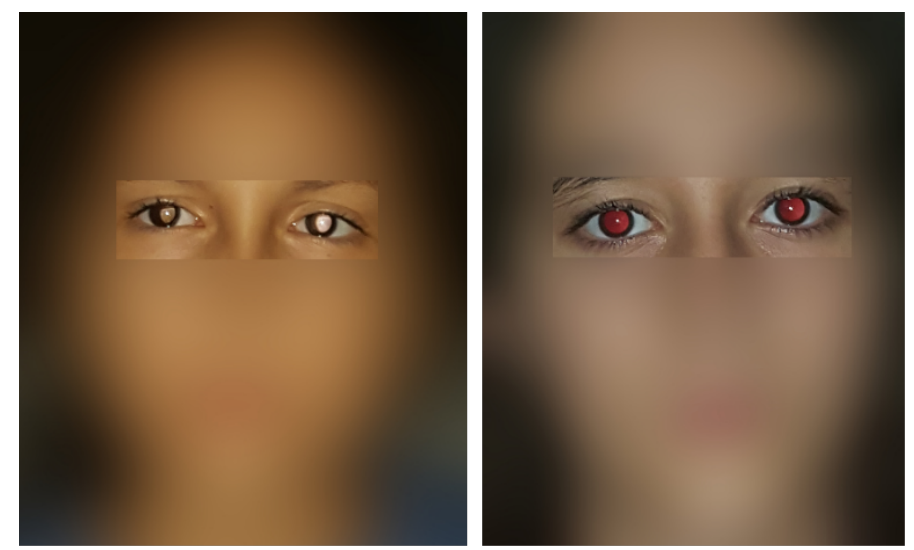

Figura 1. Exemplos da base de imagens.

Durante a aquisição das imagens, requereu-se que os pacientes estivessem com a pupila dilatada e sem óculos, pois este é o procedimento padrão do Teste de Brückner [Jalis 2015]. A base contém, no total, 58 imagens divididas em duas classes: pacientes normais (33 imagens) e pacientes com patologia (25 imagens).

\subsection{Método Proposto}

O método proposto é composto pelas seguintes etapas: (1) aquisição das imagens, (2) deteccção de olhos, (3) segmentação da região do limbo, (4) extração de características e (5) classificação. A Figura 2 apresenta o fluxo de execução de cata etapa. 


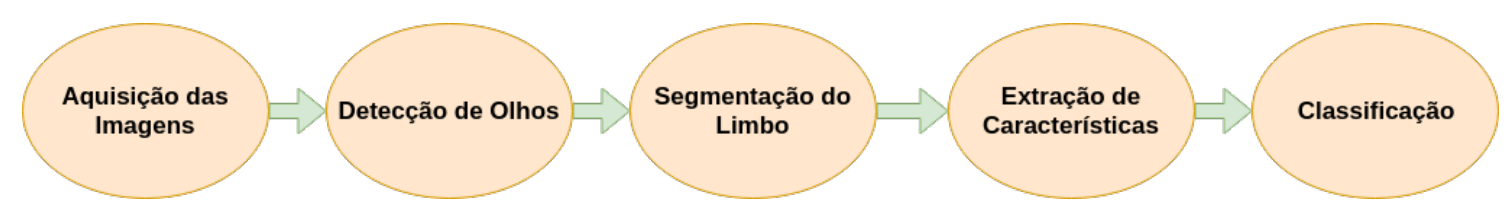

Figura 2. Metodologia proposta.

\subsection{Detecção de olhos}

As etapas do processo de detecção dos olhos do paciente podem ser visualizadas na Figura 3. Primeiro, realiza-se a delimitação da região da face utilizando a técnica Haar Cascade [Viola and Jones 2001]. Em seguida, realiza-se a detecção da região dos olhos de forma semelhante ao método de Zhang et al. (2014) que é baseado no princípio Three Court Five Eyes, o qual descreve que a face pode ser dividida em três partes iguais na vertical e em cinco, na horizontal, sendo que essas últimas têm aproximadamente a largura do olho humano.

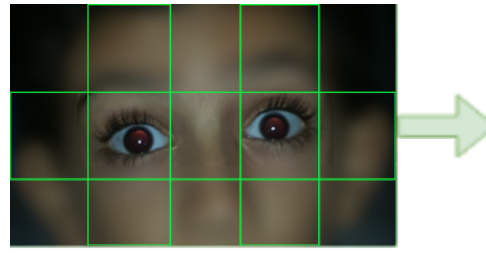

A

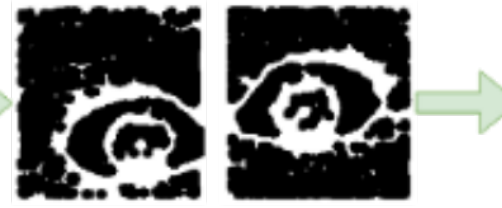

B
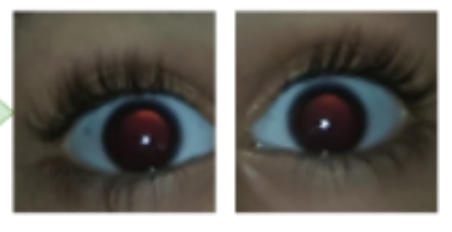

C

Figura 3. Etapas do processo de detecção de olhos.

Os olhos estão aproximadamente localizados no segundo terço da divisão vertical, e nas fatias 2 e 4 da horizontal (Figura 3.A). Essas regiões são então extraídas, convertidas para o espaço de cores HSV e submetidas à binarização por limiarização adaptativa gaussiana [Burger and Burge 2013] (Figura 3.B). Aplica-se nessa imagem binarizada o filtro da mediana (kernel 3x3) seguido das operações morfológicas de fechamento e erosão, ambas com kernels elipticos (9x9 e 5x5, respectivamente). Os baricentros dos pixels de valor 255 nas imagens em HSV são mapeados para a original em RGB e tornam-se as coordenadas centrais a partir das quais são definidas as dimensões de recorte das regiões do olhos (Figura 3.C). As dimensões escolhidas para as novas imagens foram 300x300 pixels, pois, dessa forma, é possível enquadrar cada olho por completo.

\subsection{Segmentação}

Para a compreensão da etapa de segmentação, é necessário um conhecimento a priori sobre as estruturas externas do olho humano. A Figura 4 apresenta três delas. O limbo (3) corresponde à zona de transição entre a esclera (1) e a íris (2).

A segmentação do limbo é realizada nas imagens obtidas pelo passo anterior utilizando-se o método proposto por Almeida et al. (2012) que utiliza o algoritmo de detecção de bordas de Canny e a transformada de Hough para encontrar o padrão circular da região do limbo, como pode ser visto na Figura 5.

\subsection{Extração de Características}

Nesta etapa, extraem-se as características da textura da imagem do limbo a fim de descrever o comportamento das intensidades apresentadas pelo reflexo retiniano. Para tanto, 


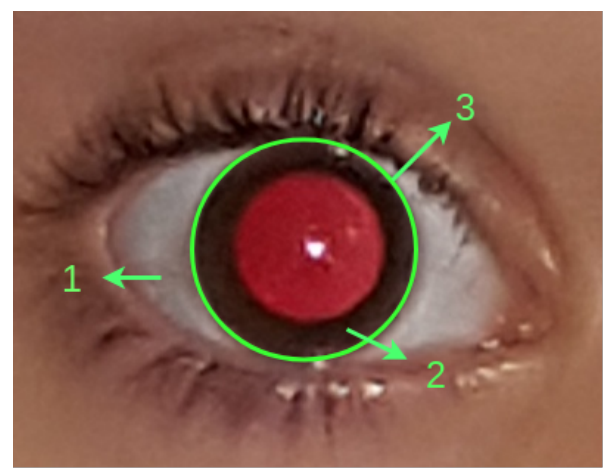

Figura 4. Regiões externas do olho humano: esclera (1), íris (2) e limbo (3).

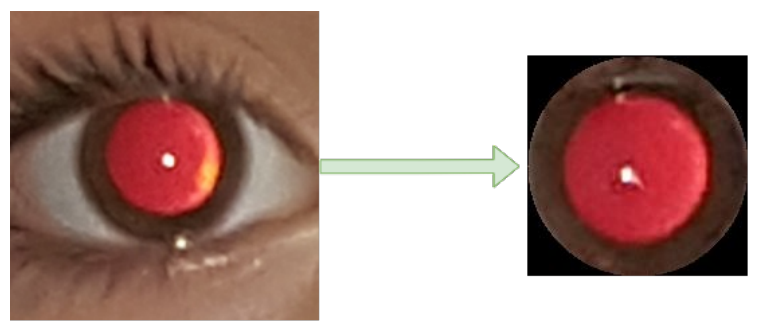

Figura 5. Segmentação do limbo.

utilizou-se os descritores de Haralick [Haralick et al. 1973], que são métricas de textura extraídas das matrizes de coocorrência de uma imagem em nível de cinza (GLCM). Essa técnica tem sido amplamente utilizada em aplicações de análise de imagens, especialmente na área da biomedicina [Zayed and Elnemr 2015]. As métricas de Haralick utilizadas no presente trabalho são: segundo momento angular, contraste, correlação, variância, homogeneidade, soma das médias, soma das variâncias, soma das entropias, entropia, variância da diferença, entropia da diferença, energia e probabilidade máxima.

No método proposto, que é aplicado em imagens RGB, as matrizes de coocorrência são calculadas separadamente nos canais $\mathrm{R}, \mathrm{G}$ e $\mathrm{B}$ nas direções $0^{\circ}, 45^{\circ}, 90^{\circ}$ e $135^{\circ}$. Para cada GLCM de um canal, são calculados os descritores supracitados. Esse cálculo gera quatro valores para cada métrica, dos quais obtem-se uma média. Cada canal é, então, representado por um vetor contendo as médias obtidas, totalizando 13 características. Esses vetores são concatenados e, dessa forma, cada imagem da região do limbo passa a ser representada por um vetor único com 39 características.

\section{Resultados e Discussão}

Neste trabalho, as etapas de detecção de olhos, segmentação do limbo e extração de características foram implementadas em linguagem Python 3.6 com auxílio da ferramenta OpenCV [Itseez 2015]. Para a etapa de classificação e a posterior avaliação dos resultados, foi utilizada a ferramenta WEKA [Hall et al. 2009] em conjunto com o Auto-WEKA [Thornton et al. 2013] .

O método de detecção de olhos obteve $100 \%$ de acerto na localização da região dos olhos nas imagens da base adquirida. A segmentação do limbo foi bem sucedida em $69 \%$ das imagens. Esse método falha na segmentação do limbo quando aplicado nas imagens em que o paciente está com os olhos entreabertos, fazendo com que a região do 
limbo perca a informação de circularidade. Nesses casos, eram geradas regiões contendo parte das pálpebras, ou apenas parte do reflexo retiniano. Assim, optou-se por descartálas das etapas posteriores. Das 58 imagens originais, 116 regiões de olho foram extraídas. Dessas, somente 80 tiveram o limbo segmentado corretamente, assim passando para as etapas de extração de características e classificação.

Conforme citado na Seção 2.5, as imagens têm suas características representadas pelos descritores de Haralick aplicados nos canais R, G e B. Na etapa de classificação, foram utilizados os classificadores REPTree, Random Forest, Máquina de vetores de suporte (SVM) e KNN. As métricas de avaliação utilizadas para avaliar o desempenho da classificação foram a acurácia, sensibilidade e especificidade. Os resultados podem ser visualizados na Tabela 1 . O treinamento e o teste das instâncias seguiram o método de validação cruzada com 10 folds. Os parâmetros dos classificadores REPTree e Random Forest foram estimados pelo Auto-WEKA. Para estimar os hiperparâmetros do SVM, utilizou-se o método GridSearch, também integrado ao WEKA.

\begin{tabular}{cccc}
\hline Classificadores & Acurácia & Sensibilidade & Especificidade \\
\hline REPTree & $\mathbf{9 1 , 0 0 \%} \pm \mathbf{1 , 6 2}$ & $\mathbf{9 0 , 9 0 \%} \pm \mathbf{2 , 3 0}$ & $\mathbf{9 1 , 1 4 \%} \pm \mathbf{1 , 2 5}$ \\
Random Forest & $86,25 \% \pm 1,97$ & $90,46 \% \pm 2,96$ & $81,12 \% \pm 3,60$ \\
SVM & $85,25 \% \pm 2,23$ & $89,54 \% \pm 2,59$ & $80,00 \% \pm 4,96$ \\
KNN(k=3) & $84,50 \% \pm 0,68$ & $94,92 \% \pm 1,15$ & $71,50 \% \pm 1,96$ \\
\hline
\end{tabular}

Tabela 1. Resultados da classificação.

Dentre os classificadores utilizados, o REPTree apresentou a melhor acurácia (91\%). A métrica de sensibilidade avalia a taxa de acerto para os olhos normais, enquanto que a especificidade verifica os acertos para os olhos com patologia. Os demais classificadores apresentaram taxa de especificidade menor que a sensibilidade. Com o REPTree acontece o contrário. A despeito da tênue diferença entre os valores dessas métricas, esse classificador discrimina melhor os casos em que o olho sofre de patologia.

\section{Conclusão e Trabalhos Futuros}

A partir dos resultados obtidos, observa-se que eles apresentam perspectivas favoráveis ao método proposto. Entretanto, para que haja uma análise mais completa e conclusões mais acuradas, é preciso que mais imagens sejam submetidas ao método.

Como trabalhos futuros, cita-se o aumento da base de imagens e a investigação de outras técnicas para melhorar a etapa de segmentação da região do limbo a fim de que não se perca imagens durante a execução do método proposto. Pretende-se, também, avaliar os resultados do método quando aplicado em imagens contendo somente a região do reflexo retiniano. Serão estudadas diferentes abordagens de extração de características de textura de forma a melhorar os resultados já obtidos. Os autores agradecem à FAPEMA (Processo: UNIVERSAL-01082/16) e ao CNPq (Processo: 423493/2016-7) pelo apoio financeiro.

\section{Referências}

Almeida, J. D. S., Silva, A. C., de Paiva, A. C., and Teixeira, J. A. M. (2012). Computational methodology for automatic detection of strabismus in digital images through hirschberg test. Computers in Biology and Medicine, 42(1):135 - 146. 
Burger, W. and Burge, M. J. (2013). Principles of Digital Image Processing: Advanced Methods. Springer Publishing Company, Incorporated.

Hall, M., Frank, E., Holmes, G., Pfahringer, B., Reutemann, P., and Witten, I. H. (2009). The weka data mining software: An update. SIGKDD Explor. Newsl., 11(1):10-18.

Haralick, R. M., Shanmugam, K., et al. (1973). Textural features for image classification. IEEE Transactions on systems, man, and cybernetics, (6):610-621.

Henning, R., Rivas-Perea, P., Shaw, B., and Hamerly, G. (2014). A convolutional neural network approach for classifying leukocoria. In Image Analysis and Interpretation (SSIAI), 2014 IEEE Southwest Symposium on, pages 9-12. IEEE.

Itseez (2015). Open source computer vision library. https://github.com/ itseez/opencv.

Jalis, M. (2015). Use of bruckner test for the detection of significant refractive errors in children. Journal of Rawalpindi Medical College, 19(3):200-203.

Rivas-Perea, P., Baker, E., Hamerly, G., and Shaw, B. F. (2014). Detection of leukocoria using a soft fusion of expert classifiers under non-clinical settings. BMC ophthalmology, 14(1):110.

Sun, M., Ma, A., Li, F., Cheng, K., Zhang, M., Yang, H., Nie, W., and Zhao, B. (2016). Sensitivity and specificity of red reflex test in newborn eye screening. The Journal of pediatrics, 179:192-196.

Thornton, C., Hutter, F., Hoos, H. H., and Leyton-Brown, K. (2013). Auto-weka: Combined selection and hyperparameter optimization of classification algorithms. In Proceedings of the 19th ACM SIGKDD international conference on Knowledge discovery and data mining, pages 847-855. ACM.

Viola, P. and Jones, M. (2001). Rapid object detection using a boosted cascade of simple features. In Computer Vision and Pattern Recognition, 2001. CVPR 2001. Proceedings of the 2001 IEEE Computer Society Conference on, volume 1, pages I-I. IEEE.

Zayed, N. and Elnemr, H. A. (2015). Statistical analysis of haralick texture features to discriminate lung abnormalities. Journal of Biomedical Imaging, 2015:12:12-12:12.

Zhang, L., Lu, K., Pan, C., and Xia, S. (2014). Eye detection for electronic map control application. In Intelligent Human-Machine Systems and Cybernetics (IHMSC), 2014 Sixth International Conference on, volume 1, pages 241-244. IEEE. 